FINAL REPORT

\title{
IMPROVEMENT OF PULPING UNIFORMITY BY MEASUREMENT OF SINGLE FIBER KAPPA
}

\author{
DOE CONTRACT OR GRANT NUMBER: DE-FC07-97ID13539
}

RICHARD R. GUSTAFSON AND JAMES B. CALLIS

\author{
UNIVERSITY OF WASHINGTON
}

\begin{abstract}
$\underline{\text { Abstract }}$
A method to measure the kappa of single fibers by staining with a fluorescent dye, Acridine Orange (AO), has been developed. This method is now applied to develop an automated flow-through instrument that permits routine kappa analysis on thousands of fibers in a pulp sample. The instrument can reliably collect and process fluorescent images of AO stained fibers to give the fiber kappa number distribution of a pulp sample in a few minutes. The design and operation of the instrument are similar to that of a flow cytometer but with the addition of extensive fiber imaging capability. Fluorescence measurements in the flow-through instrument are found to be consistent with those made with a fluorescence microscope provided the signal processing in the flow-though instrument is handled properly. The kappa distributions of pulps that were analyzed by means of a density gradient column are compared to those measured with the flowthrough instrument with good results. The kappa distributions of various laboratory pulps and commercial pulps have been measured. It has been found that all pulps are non-uniform but that commercial pulps generally have broader kappa distributions than their laboratory counterparts. The effects of different pulping methods and chip pretreatments on pulp uniformity are discussed in the report. Finally, the application of flow-through fluorescence technology to other single fiber measurements are presented
\end{abstract}




\section{Introduction}

Uniform pulp has many benefits for the economic and environmental performance of the pulping industry. Process improvements promoting uniformity enabled extended delignification, lowering bleaching costs, and improving environmental performance [1]. More uniform pulp also has superior strength delivery [2], although this improvement may not be observed in some bleached pulps [3]. High yield pulping also benefits from uniformity improvements which result in fewer knotter rejects at a given kappa [4]. Lower reject levels also allow an increase in the target kappa, shortening cook times and improving digester throughput.

Measurement of pulp uniformity at the fiber level began in the 1970's. Paulson used a density gradient column to measure the lignin content distributions of thin and thick chips as well as chips from compression wood [5]. The density gradient column separation takes advantage of the fact that lignin and cellulose have different densities. Fibers will migrate to the section of the column that matches their density. Recently, Rudie and Boyer compared mill and laboratory pulps in a density gradient column and found that mill pulps tend to be less uniform than laboratory produced pulps [6]. They also used Fourier Transform Infrared spectroscopy to validate the density gradient method by showing that both methods produce similar fiber kappa distributions.

A new method to measure pulp uniformity has been developed at the University of Washington [7]. This method uses the fluorescence of Acridine Orange stained fibers to measure the kappa number. After screening a large number of fluorescent stains as lignin indicators, Acridine Orange (AO) showed a noticeable color shift in the presence of lignin. The ratio of the spectral peak height of the dimer form of AO (red) to the peak height of the monomer form of AO (green) was shown to correlate linearly with lignin content. Using this red to green ratio, measurements of lignin content distributions were made under a fluorescence microscope.

Using the AO fluorescence microscope method, kappa distributions for many pulps were measured, revealing nonuniformity even in pulps carefully prepared with thin chips and mild pulping conditions [8].

While the Acridine Orange fluorescence provides a useful method for measuring fiber kappa numbers, it has some limitations. First, the method is slow to run with a fluorescence microscope. Only one fiber at a time can be scanned, thus only twenty or thirty fibers may be analyzed in an hour; hardly sufficient to construct a fiber kappa histogram. In addition, the calibration curve relating fiber kappa to red/green ratio is not universal. Wood species and the presence of significant quantities of reprecipitated lignin may change the slope of the calibration between kappa and the ratio of red fluorescence to green fluorescence.

To be used for routine analysis, the AO fluorescence method must be modified for use in a flow through analysis apparatus. Fluorescence is the ideal spectroscopic method to be used in flow through analysis systems because of its large signal to noise ratio. The objective of this research then was to develop and apply a flow-through fluorescence image analyzer that can use the fluorescence of Acridine Orange stained fibers to measure single fiber kappa numbers. Applications of the instrument include investigating the effect of chip properties and digester configurations on pulp uniformity and improving the AO fluorescence method (e.g. the effects of wood species on the calibration curve) by allowing many pulps to be thoroughly analyzed in a reasonably 
short period of time. The following paper describes the development and evaluation of the instrument, and some preliminary analyses of laboratory and commercial pulps.

\section{Apparatus}

The flow-through fluorescence image analyzer, or Fiber Kappa Analyzer (FKA) consists of a hydraulic section, an optical section, and a data processing section. These function together to deliver fibers to a sensing zone, to capture images of their fluorescence, and to quantify red and green fluorescence from the captured images.

Figure 1 shows a flow diagram for the FKA's hydraulic section. The AO stained fiber sample slurry is mixed with dilute sodium hypochlorite from gear pump 1 just before it reaches the flow cell. Hypochlorite reacts with the Acridine Orange in solution and reduces background fluorescence in the image, which would otherwise interfere with a quantitative measurement of fiber fluorescence intensity. The flow cell is made from a $500 \mathrm{~m}$ soft polyethylene gasket sandwiched between a plexiglass back and a glass window on the front of the cell. The fibers are filtered out of the stream after the flow cell, and the total flow is pumped out with gear pump 2 at approximately $40 \mathrm{ml} / \mathrm{min}$. A tank following gear pump 2 ensures that air will not back up into the pump and cause it to lose its prime.

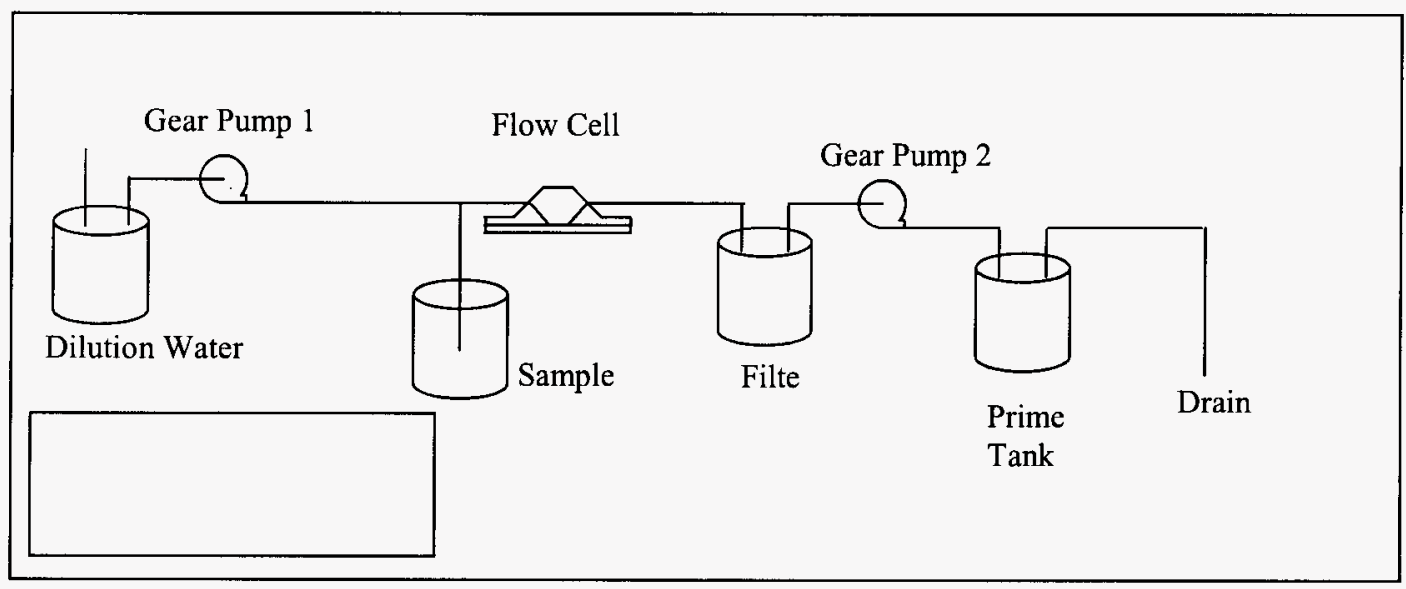

Figure 1. Flow-through instrument flow diagram

The optical section of the instrument is shown in Figure 2 and resembles a modern epi-illumination fluorescence microscope. Blue light from a 5 Joule xenon strobe passes to a dichroic mirror, where it reflects onto the AO stained fibers. The stimulated fluorescence ranging from green to red wavelengths is collimated by the objective lens and transmits through the first dichroic. A second dichroic mirror splits green and red light. Red light passes through a $590 \mathrm{~nm}$ long pass filter to a CCD camera, and green light passes through a $510 \mathrm{~nm}$ to $570 \mathrm{~nm}$ bandpass filter. Both cameras are fitted with video lenses to focus the collimated light into a fluorescence image on the CCD sensor. 


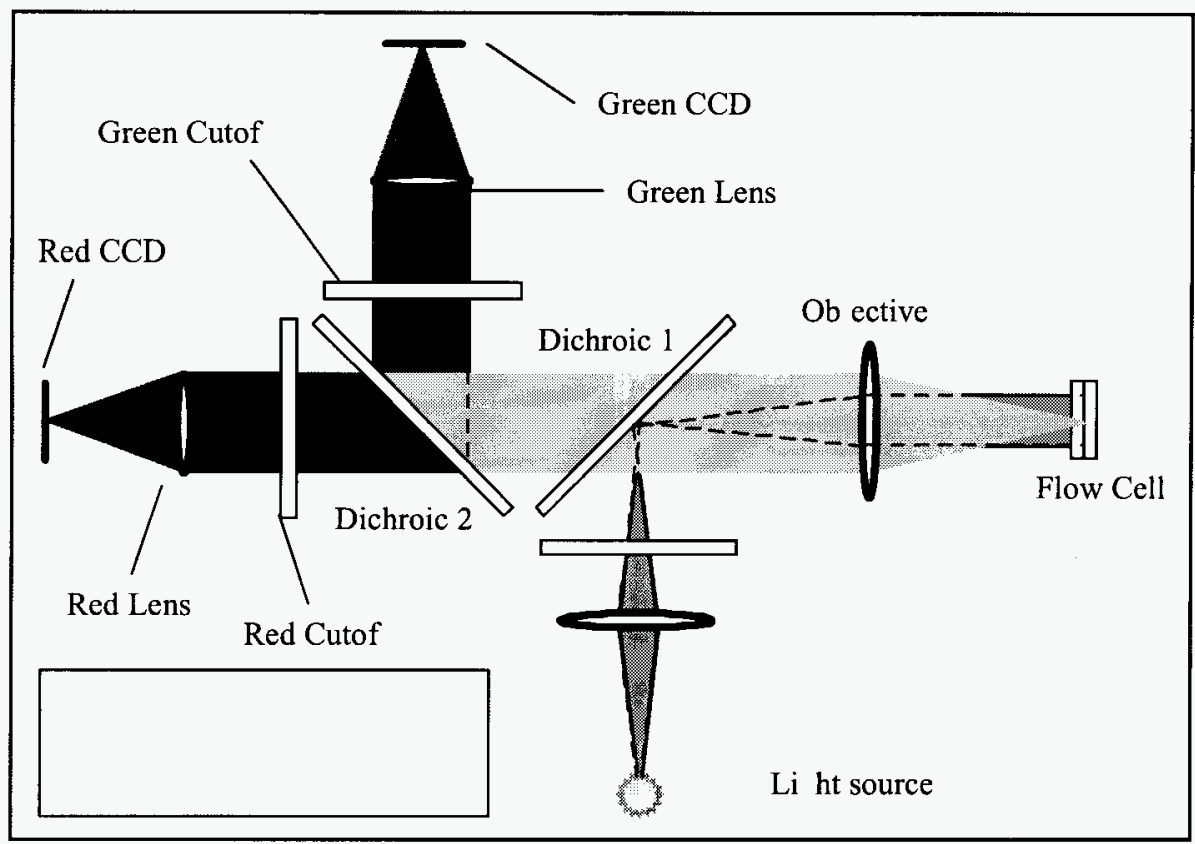

Figure 2. Flow-through instrument optical diagram

A PC fitted with frame-grabber boards and a data acquisition card initiates the capture process, and a custom made timing box synchronizes the cameras with the flashlamp and frame-grabbers. Once the images are transferred to the PC, National Instruments LabView software handles data processing. The raw fluorescence images are flattened to correct for vignetting, and the fluorescent background is removed. A binary threshold locates objects in the field, and morphological processing identifies fiber clumps and single fibers. The red and green fluorescence is calculated for each fiber and the data is cataloged into an array.

We are now building a new Single Fiber Kappa Analyzer, similar to the prototype instrument discussed above. The optics of the new instrument are being modified to improve the signal-to-noise ratio, especially when we analyze higher kappa pulps. The new instrument also has better data processing capabilities, hardware and software, so we anticipate being able to make more fiber measurements for a single pulp and be able to process more pulps in a day. We will be using this new instrument mainly for more routine analysis (as opposed to methodology development) of commercial and laboratory pulps.

\section{Analysis Procedure}

Analysis of wood fibers with the flow-through instrument first requires instrument alignment, calibration, and sample preparation. Using a slide with an image of a grid, backed with fluorescent paper, the red and green images are aligned to within a pixel. Following alignment, a calibration is performed using a fluorescence standard. The calibration corrects for uneven illumination of the field as well as vignetting in the images and fluctuations in camera gains. The resulting calibrated images are flattened and have a reproducible response to red and green fluorescence. The pulp sample is 
prepared in a liter of $5 \times 10^{-5} \mathrm{M}$ Acridine Orange at a consistency of $0.01 \%$. The slurry is blended for five seconds with a commercial Waring blender to separate fiber clumps. The pulp is allowed to react for at least 10 minutes with the Acridine Orange before it is analyzed in the FKA.

\section{$\underline{\text { AO safety and handling }}$}

A review of the literature found the following information on use of Acridine Orange. It should be noted that Acridine Orange is a very common stain used safely in many laboratories around the world.

\section{Health Hazard Data}

(Sources: Molecular Probes MSDS and Fisher Scientific MSDS sheet.)

https://fscimage.fishersci.com/msds/86497.htm

Possible routes of entry are inhalation, skin adsorption and ingestion. AO is not reported to be carcinogenic but is listed on some MSDS sheets as mutagenic. The effects of AO exposure are irritation if swallowed, inhaled, or absorbed through skin. No other effects of overexposure have been documented. The toxicity data indicate a $\mathrm{LD}_{50}$ of 64 $\mathrm{mg} / \mathrm{kg}$, in mice. The effects of exposure to Acridine Orange solution that have been reported are that it is mildly toxic and can cause irritation. The concentrations that are required to conduct the analyses in this work $\left(10^{-5} \mathrm{M}\right)$ are too low to have a harmful affect even if the chemical is handled improperly.

\section{Acridine Orange in the paper product or the waste stream}

We considered a worst-case scenario to see if there should be concern with Acridine Orange getting into the paper or effluent stream. If Acridine Orange was used regularly ( 100 measurements per day) a six months supply would weigh about 30 grams. If all of this were dumped into the wet end of the paper machine the concentration of AO in the product (assuming it all ends up in the sheet) for the hour or so you might see it averages $0.7 \mathrm{ppm}$. This is of course a very rough calculation. Our understanding of AO toxicity suggests that at this concentration the paper would not present a health hazard.

For the effluent calculation, if you dump all 30 grams into the waste stream, over the next hour you might see an AO concentration of $10^{-8}$ or $10^{-9} \mathrm{M}$, which is near the detection limit.

The results of our literature search and worst case scenario calculations show that Acridine Orange could be used safely in a mill environment if normal precautions are taken.

\section{Instrument Validation}

Uniform fluorescent polystyrene beads manufactured as standards for flow cytometry were used to assess the performance of the Single Fiber Kappa Analyzer (FKA). Uniform fibers would be preferable, but no fiber source has been identified with sufficient uniformity in the fluorescence spectrum to evaluate the instrument's performance. The signal to noise ratio $(\mathrm{s} / \mathrm{n})$ measurement from the uniform standards can be used to estimate the FKA's precision in the $R / G$ measurement of a wood fiber. Figure 3 shows the measured $\mathrm{R} / \mathrm{G}$ distribution of uniform $25 \mathrm{~m}$ beads flowing through the FKA. The mean particle intensity of these beads is approximately the same as an average stained fiber. The coefficient of variation $(\mathrm{CV})$ of the $\mathrm{R} / \mathrm{G}$ ratio for the flowing beads is 
$6.5 \%$, while the CV of a single bead repeatedly measured in one position is $3.2 \%$. For a larger particle, such as a fiber, the random errors decrease due to an increased number of pixel samples. The $\mathrm{R} / \mathrm{G} \mathrm{CV}$ for a single fiber repeatedly measured in one position is $0.49 \%$. Applying the assumption that variance is additive, a CV of $5.5 \%$ can be estimated for a uniform fiber source, which corresponds to an uncertainty of 2 kappa units for a kappa 30 fiber. This low uncertainty guarantees that the variance in the instrument contributes negligibly to the variance measurements of the pulp samples.

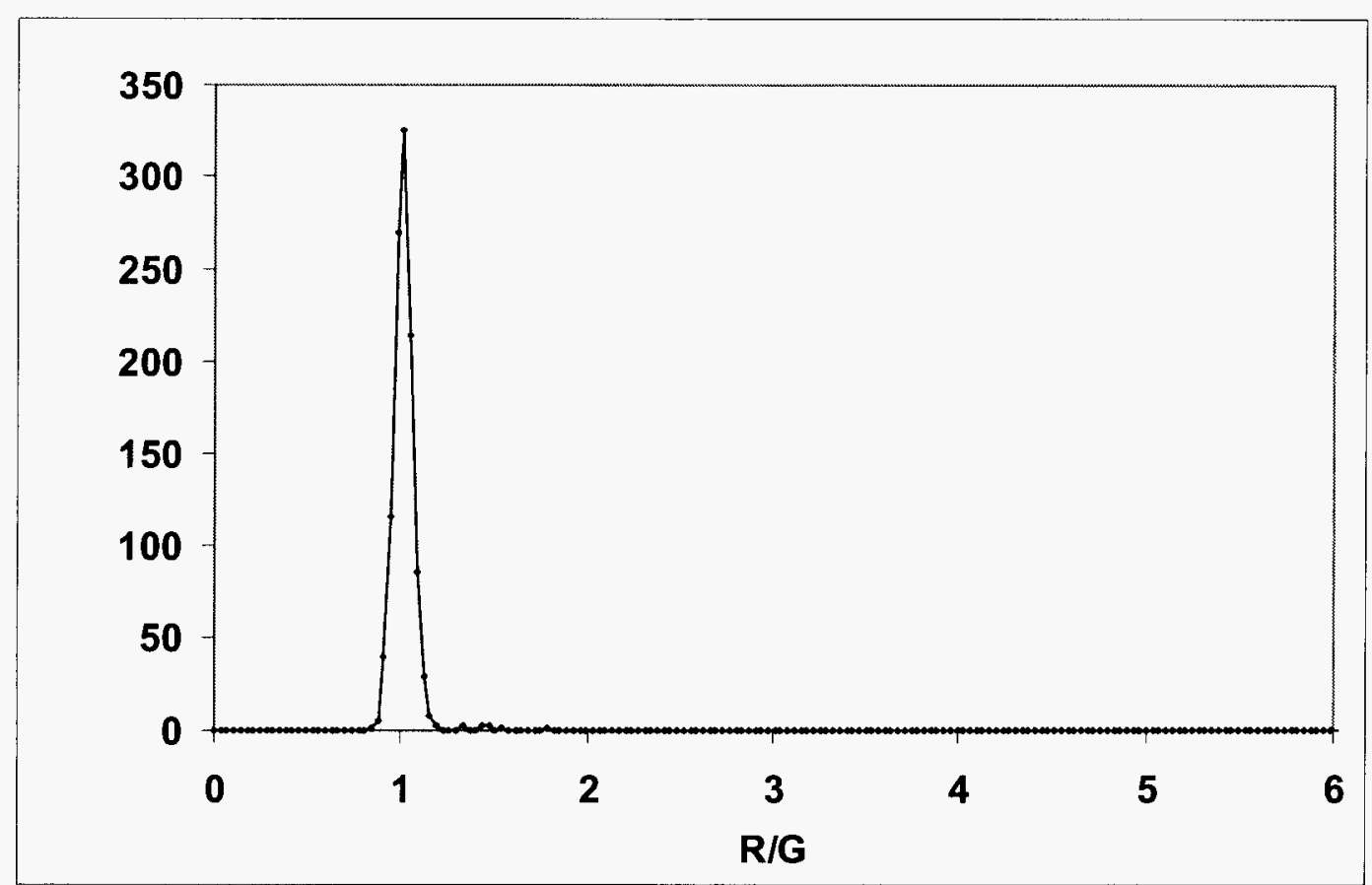

Figure 3. Red/Green distribution for 25 micrometer Nile Red beads flowing through single fiber analyzer

\section{Comparison with Microscope Method}

Uniform laboratory pulps were previously prepared for development of the AO fluorescence method performed with a fluorescence microscope [9]. These samples were analyzed with the FKA in order to verify that earlier results could be reproduced with the new instrument. Figure 4 compares the distributions of two representative pulps measured on the FKA with those measured with the fluorescence microscope method. The microscope distribution is noisy (roughly fifty fibers were measured per sample), but the resolution is sufficient to illustrate that the distributions match fairly well. 


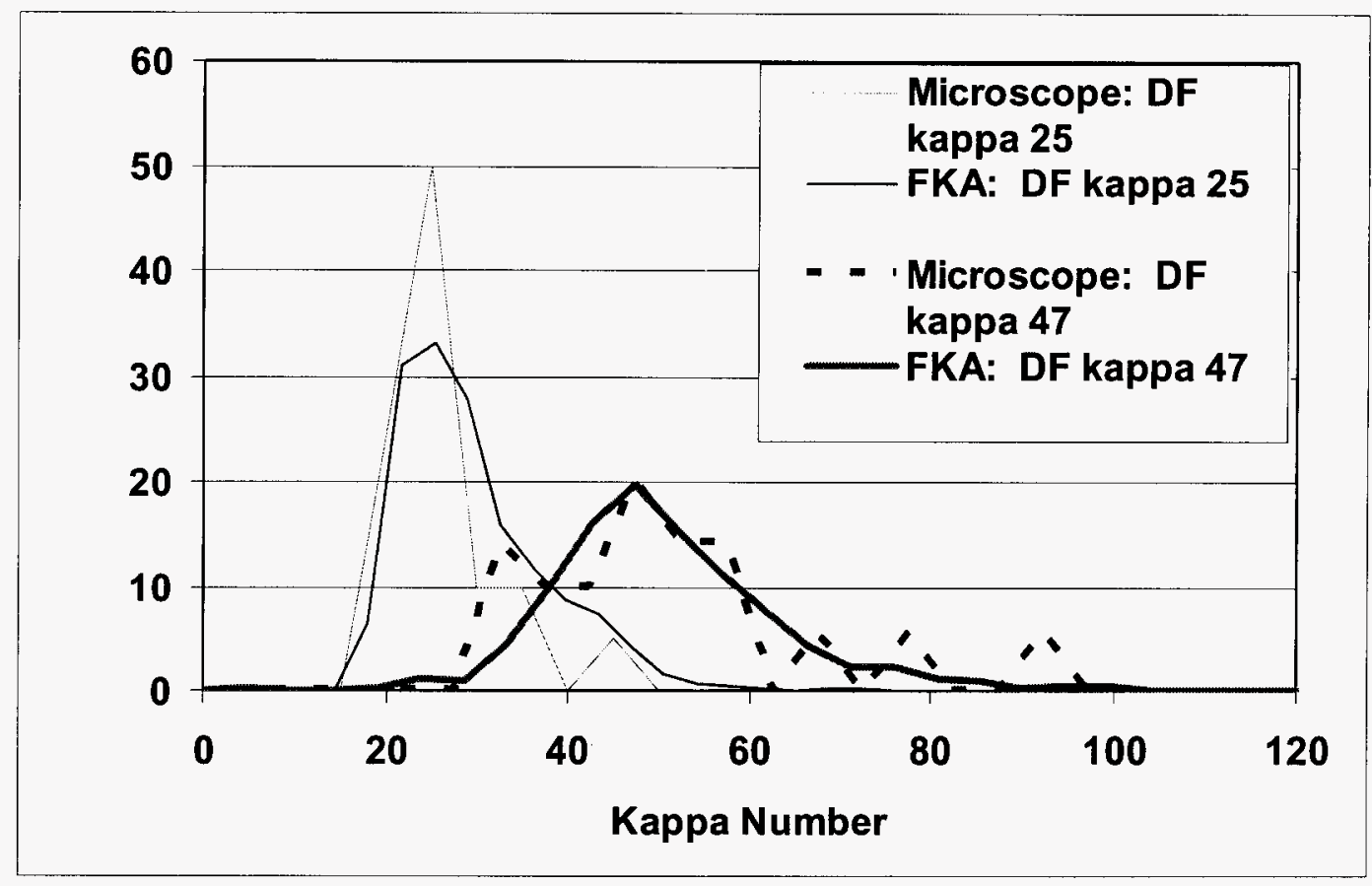

Figure 4. Comparison of Kappa distribution measured on flow-though apparatus and with fluorescence microscope.

Validation with Independent Samples

Pulp samples prepared and analyzed on a density gradient column by Alan Rudie and Brian Boyer at the Institute of Paper Science and Technology (IPST) were analyzed with the FKA. These IPST pulps were produced in cooks with a liquor to wood ratio of $6: 1$, sulfidity of $30 \%$ and a $24 \%$ EA charge. The kappa distributions of the IPST pulps measured with the FKA and the density gradient column are shown in Figures 5 -9.

The pulps in Figures 5 and 6 were prepared from $2.5 \mathrm{~mm}$ thick Southern Pine chips. Cook 4 (Figure 5) was pulped at $165 \mathrm{C}$ to an $\mathrm{H}$-factor of 1892. Cook 9 (Figure 6) was pulped at $154 \mathrm{C}$ to an $\mathrm{H}$-factor of 786 [10]. Error estimates for the FKA sample in Figure 6 reflect Poisson counting statistics. Figures 5 and 6 show that analyses performed with the FKA produce similar results to those conducted with a density gradient column. 


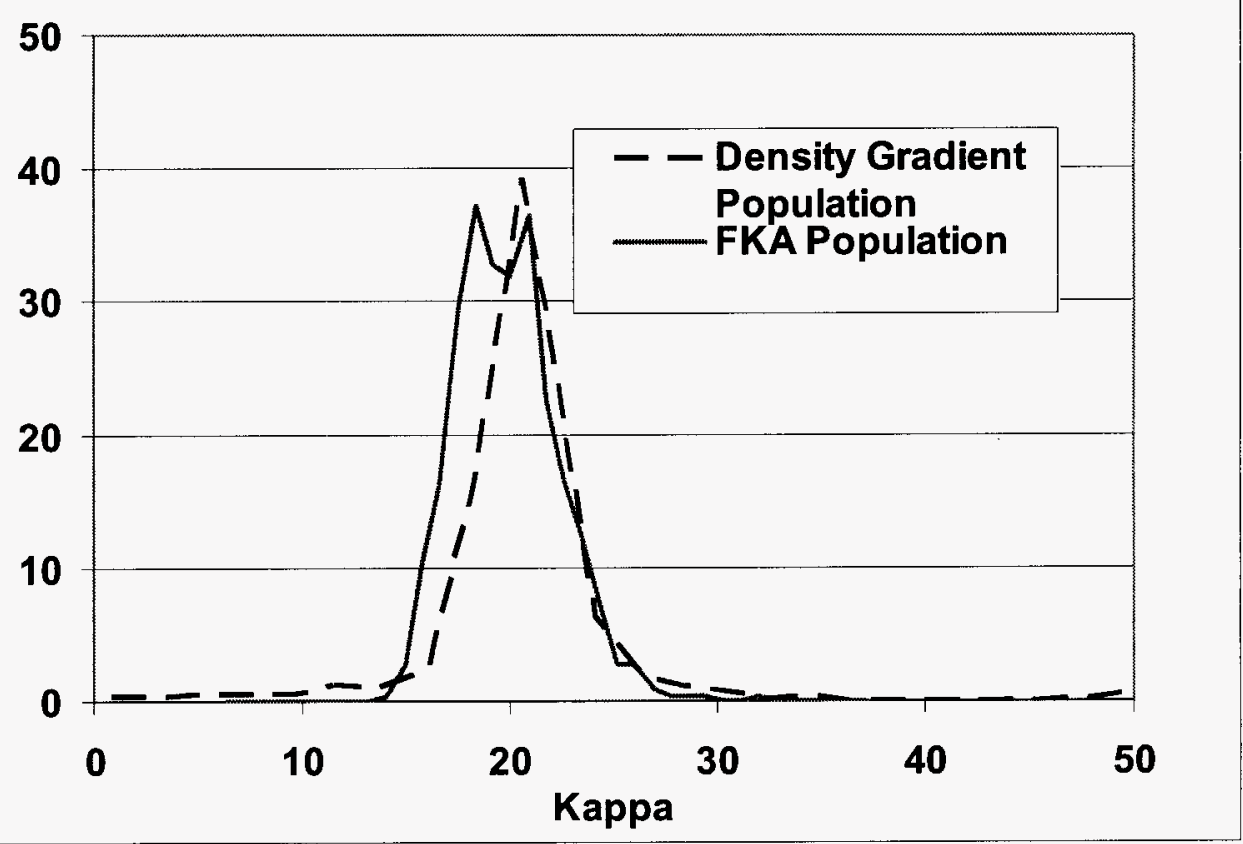

Figure 5. Comparison of density gradient and FKA for a kappa 15.9 uniform pulp. IPST cook 4.

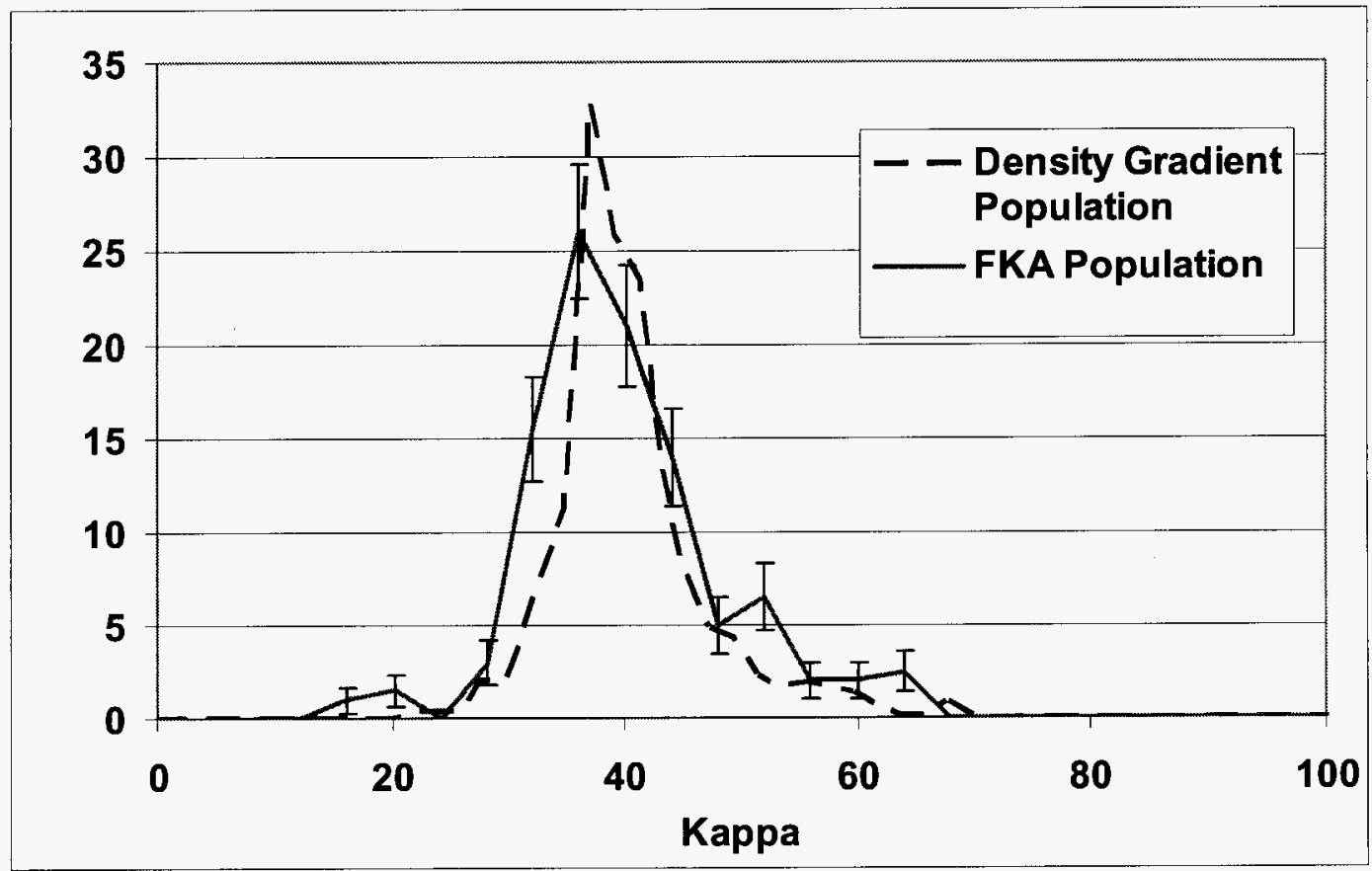

Figure 6. Comparison of density gradient and FKA for a kappa 33.9 uniform pulp. IPST cook 9. 
The pulps in Figures 7 and 8 were prepared at IPST from $10 \mathrm{~mm}$ thick chips. Cook 3 (Figure 7) was pulped at $170 \mathrm{C}$ to an $\mathrm{H}$-factor of 2803, and cook 7 (Figure 8) was pulped at $160 \mathrm{C}$ to an $\mathrm{H}$-factor of 1274 [10]. These thick chip pulps have a wider kappa distribution than the thin chip pulps shown in Figures 5 and 6 . Error estimates for the FKA sample in Figure 8 reflect Poisson counting statistics. For the nonuniform pulps, the FKA and the density gradient column show the presence of a high kappa tail, and both measured roughly the same kappa distribution.

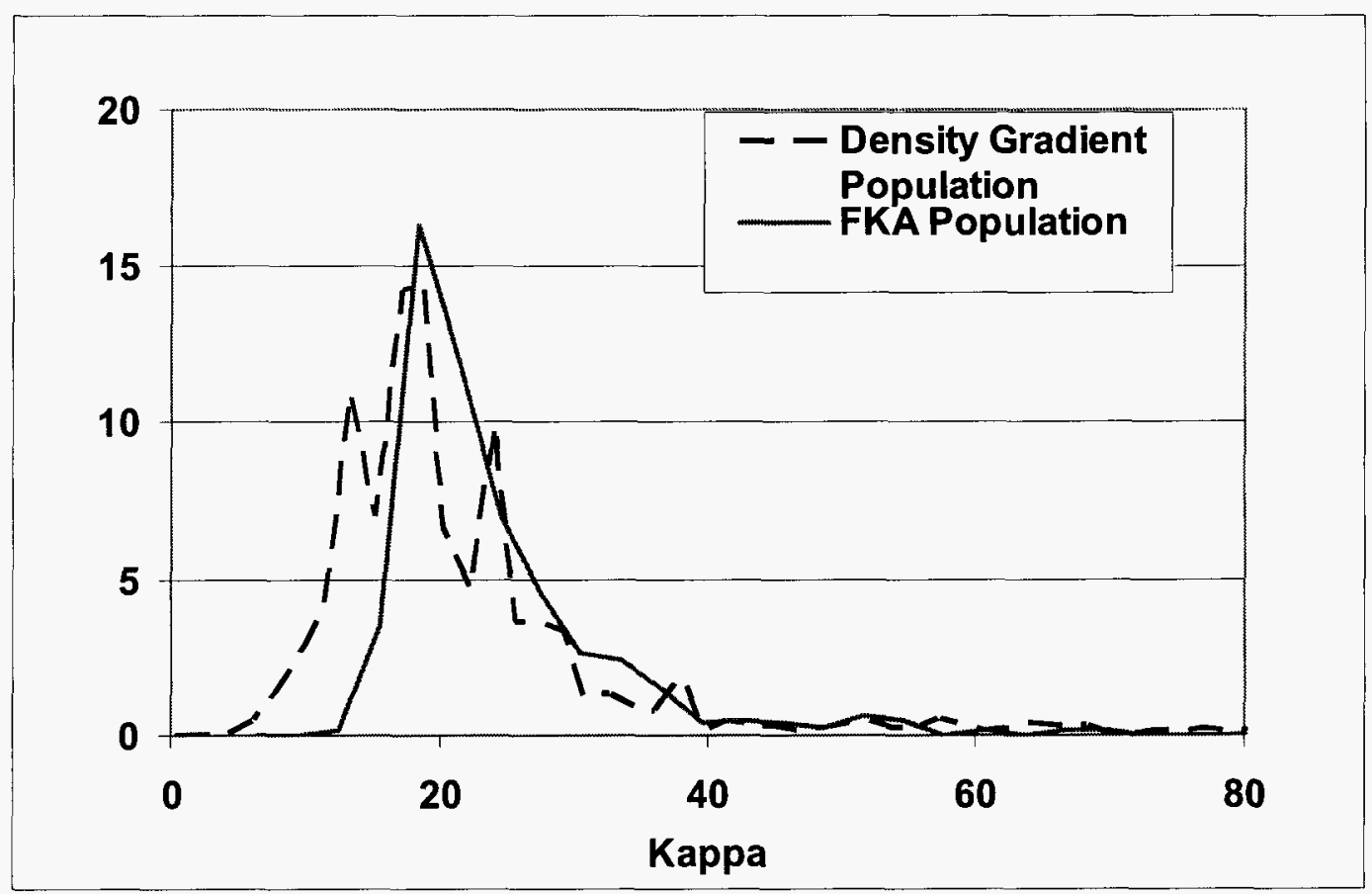

Figure 7. Comparison of density gradient and FKA for a kappa 23.4 non-uniform pulp. IPST cook 3 . 


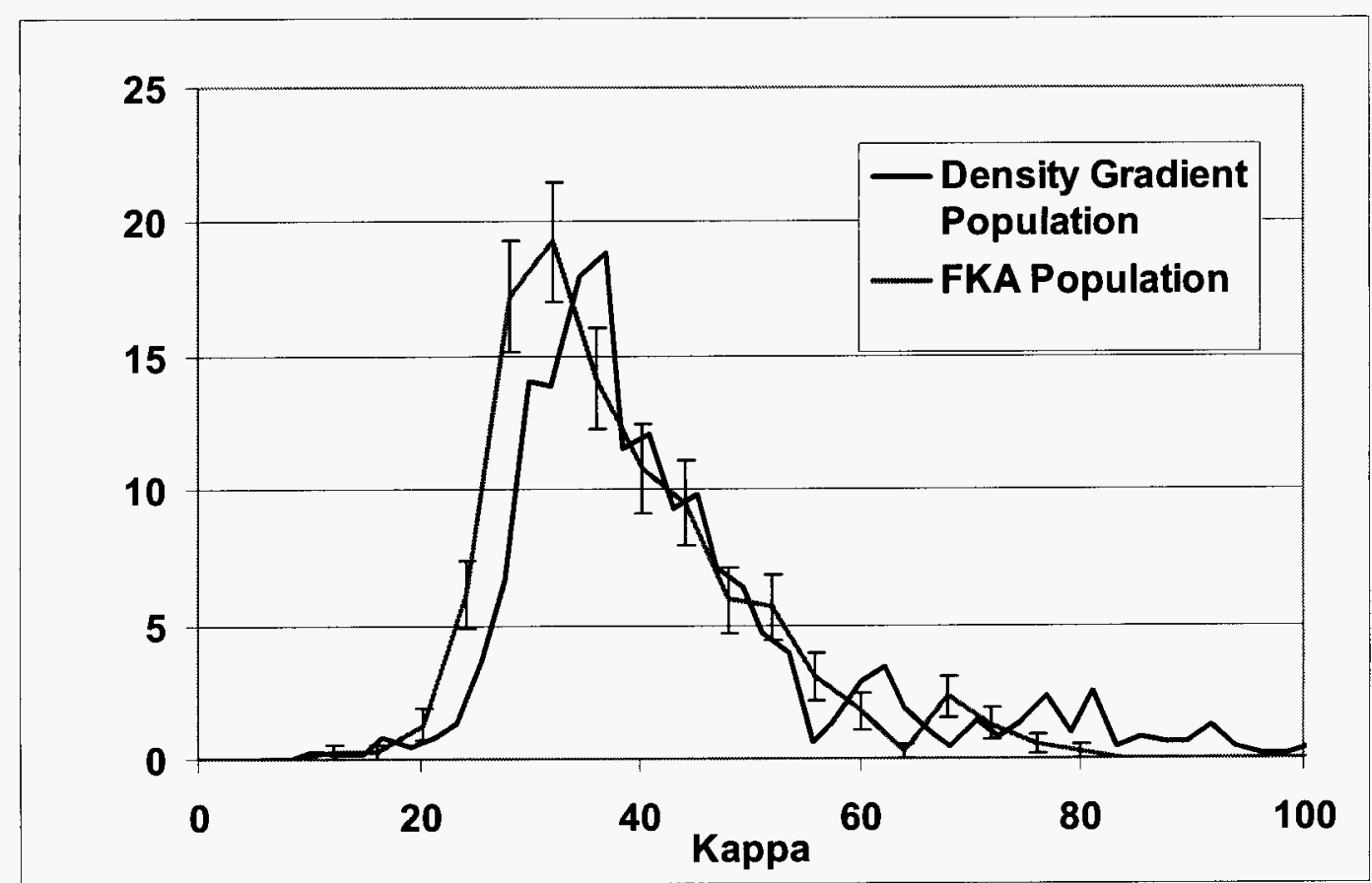

Figure 8. Comparison of density gradient and FKA for a kappa 33.4 non-uniform pulp. IPST cook 7.

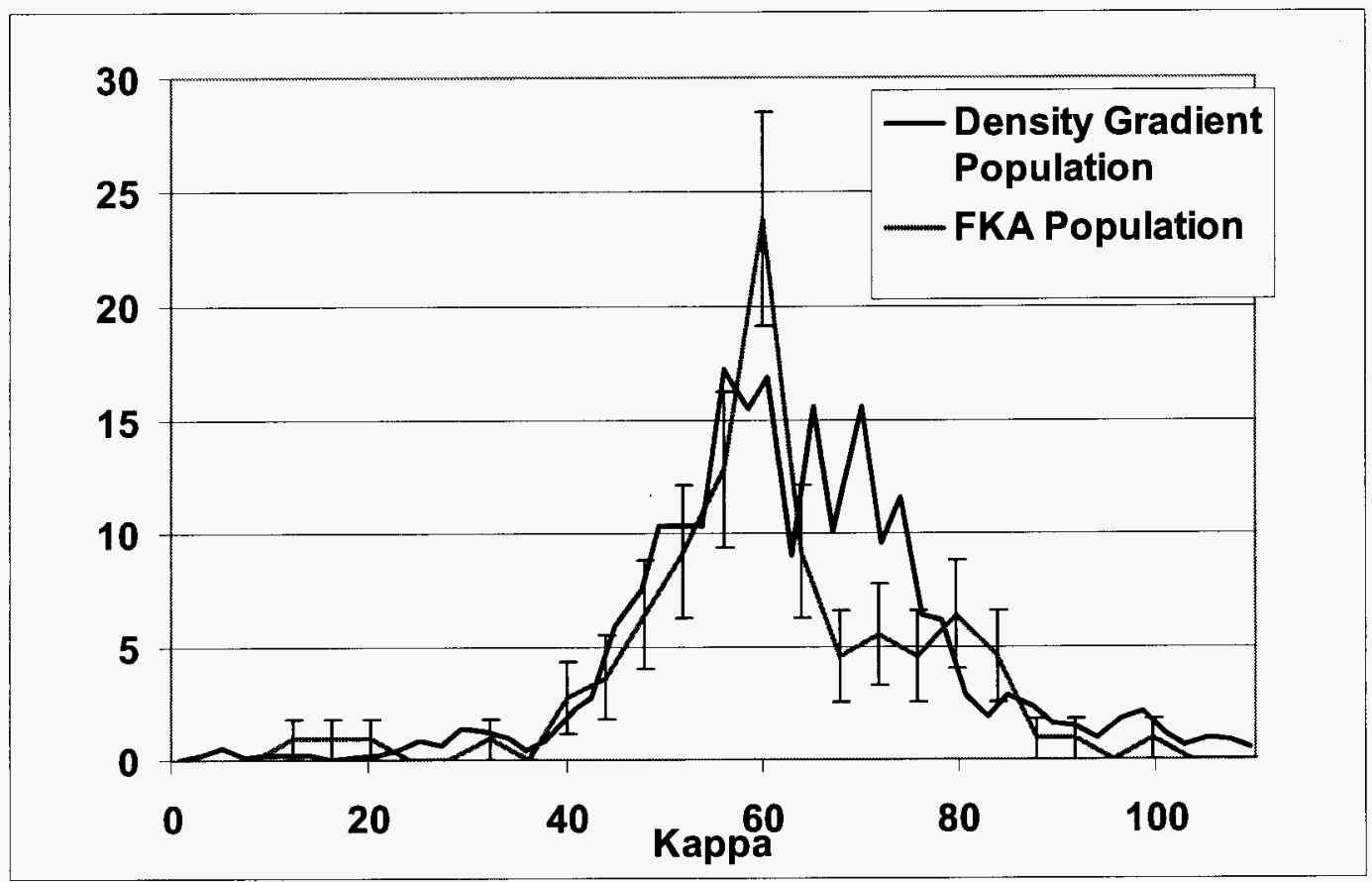

Figure 9. Comparison of density gradient and FKA for a kappa 63.7 pulp. IPST cook 1. 
In Figure 9, a high kappa pulp from $10 \mathrm{~mm}$ thick chips is shown. This pulp was cooked at $150 \mathrm{C}$ to an $\mathrm{H}$-factor of 548 [10]. Again, the density gradient column and the FKA measure similar distributions for this pulp within experimental error.

\section{Analysis of Laboratory Pulps}

The favorable comparison of the kappa distributions measured by the FKA and density gradient column validates the accuracy flow-through analyzer. The instrument has since been applied to measure the kappa uniformity of various laboratory pulps. Some cases of interest are presented here.

\section{Chip thickness study}

The first set of pulps (provided by a corporate research facility) included softwood chips segregated by size and pulped separately under identical pulping conditions. The samples were pulped at a temperature of $165 \mathrm{C}$ with an EA charge of $14.5 \%$. The liquor to wood ratio was 3.5 , and the sulfidity was $27.5 \%$. A control sample with the original chip size distribution was also pulped. Based on a diffusion limited pulping model, all the pulps should look similar at the low kappa end, but the thick chips should have more high kappa fibers, which come from the chip center. Figure 10 shows the fiber kappa histograms for the thickness study. The kappa distributions in Figure 10 behave as expected. The low target kappa for these pulps explains why the differences between chip classes are not larger. At the final average kappa of 19, many of the fibers on the low kappa side of the distributions are in a residual delignification stage, causing the distributions to narrow. Figure 11 shows that the control fiber kappa distribution can be predicted from the weighted sum of the different chip classes, adding credibility to the distributions shown in Figure 10. 


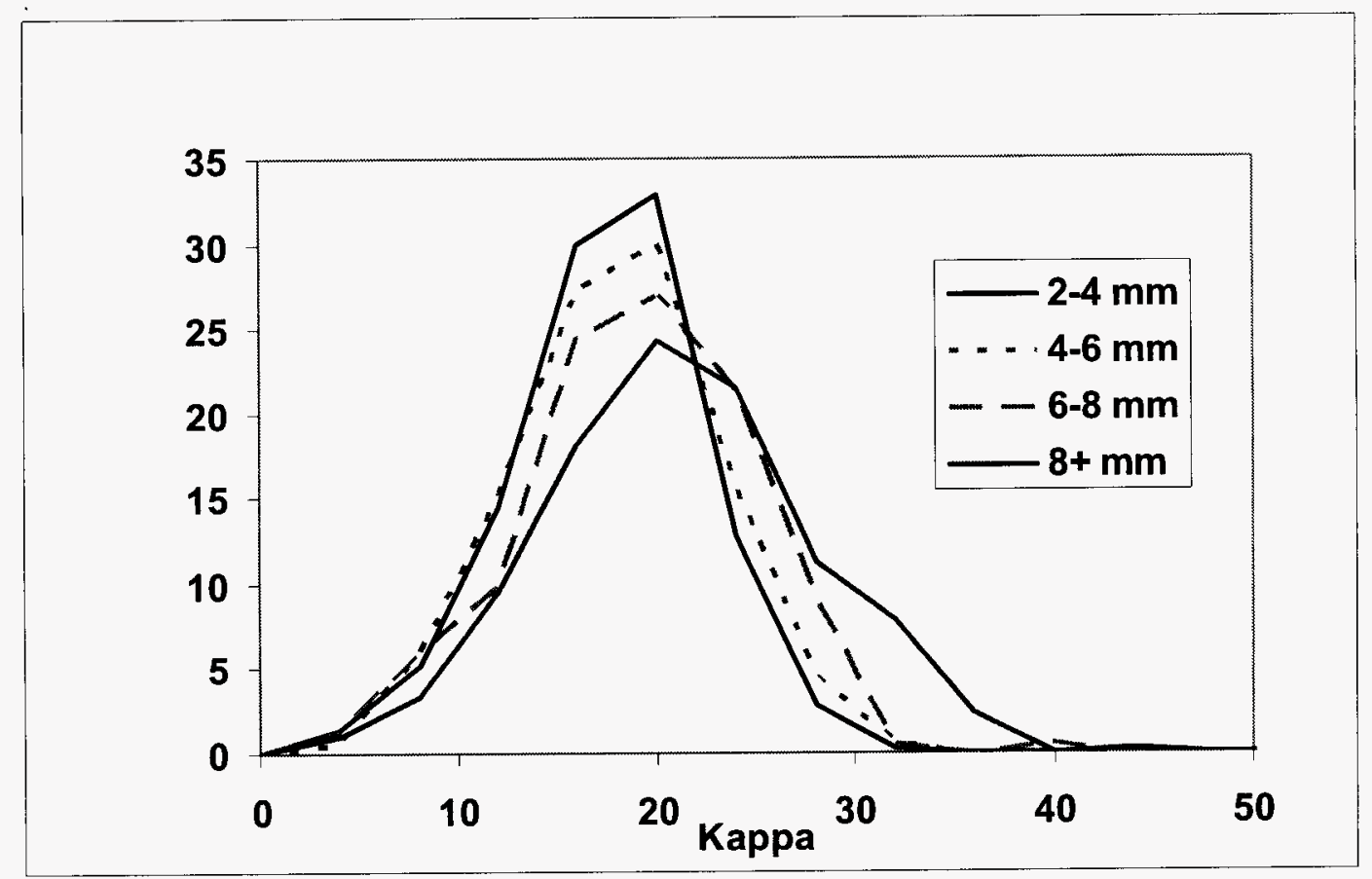

Figure 10. Fiber kappa histograms for pulps from varying chip sizes.

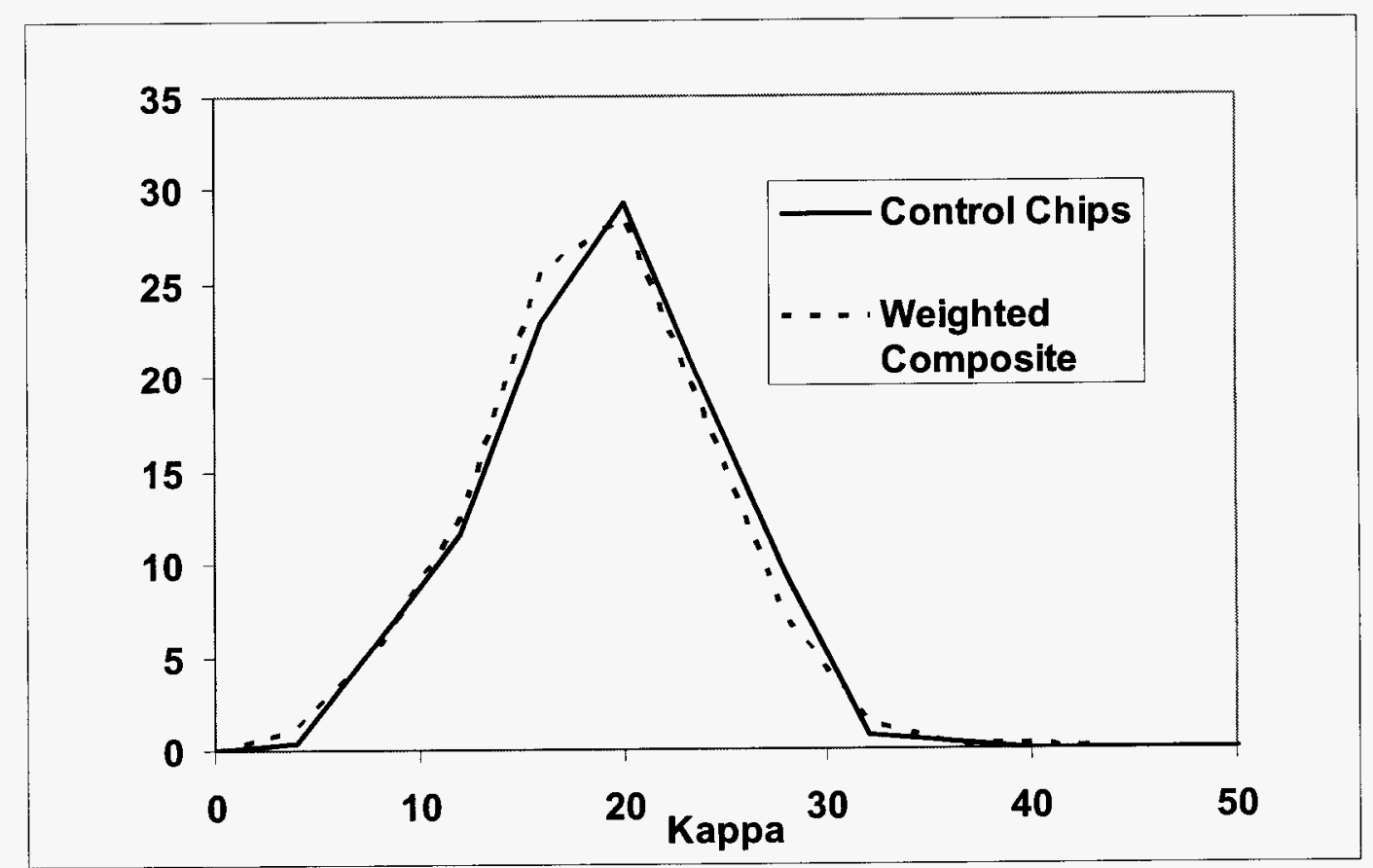

Figure 11. Comparison of measured kappa distribution and composite of separate chip size kappa distributions 
Comparison of Conventional, EMCC, EMCC-AQ pulping

Another set of pulps analyzed by the FKA were also provided by a corporate research facility. Softwood pulps were prepared by a laboratory batch digester configured to simulate EMCC, EMCC-AQ, and conventional kraft cooking with typical commercial pulping conditions. The distributions of representative kappa 22 pulps from each digester configuration are shown in Figure 12. Surprisingly, no significant differences in uniformity were observed between these pulps. If we investigate lower kappa pulps, however, some differences in the uniformity that result from these different pulping technologies may be seen. Figure 13 shows the relationship between coefficient of variation $(\mathrm{CV})$ of the single fiber kappa distribution and average kappa for the three cooking configurations. In the region between kappa 22 and kappa 28 , the CV is about the same for all three cook types, hence the similarities of the kappa distributions at kappa 22. Below this kappa region, the CV increases for EMCC-AQ pulping, while it stays fairly constant for conventional and EMCC pulps. At this late stage in pulping, a constant $\mathrm{CV}$ results from slowing delignification in low kappa fibers. An increasing CV suggests that low kappa fibers are still experiencing fairly rapid delignification. The presence of AQ in the EMCC-AQ cook may result in more reactive lignin at the end of the cook. McDonough and Herro previously found a similar result investigating the effect of AQ on bleachability, and they hypothesized that AQ inhibited lignin condensation and/or bond formation between lignin and carbohydrates during the cook, thus improving the reactivity of lignin remaining in a low kappa pulp [11].

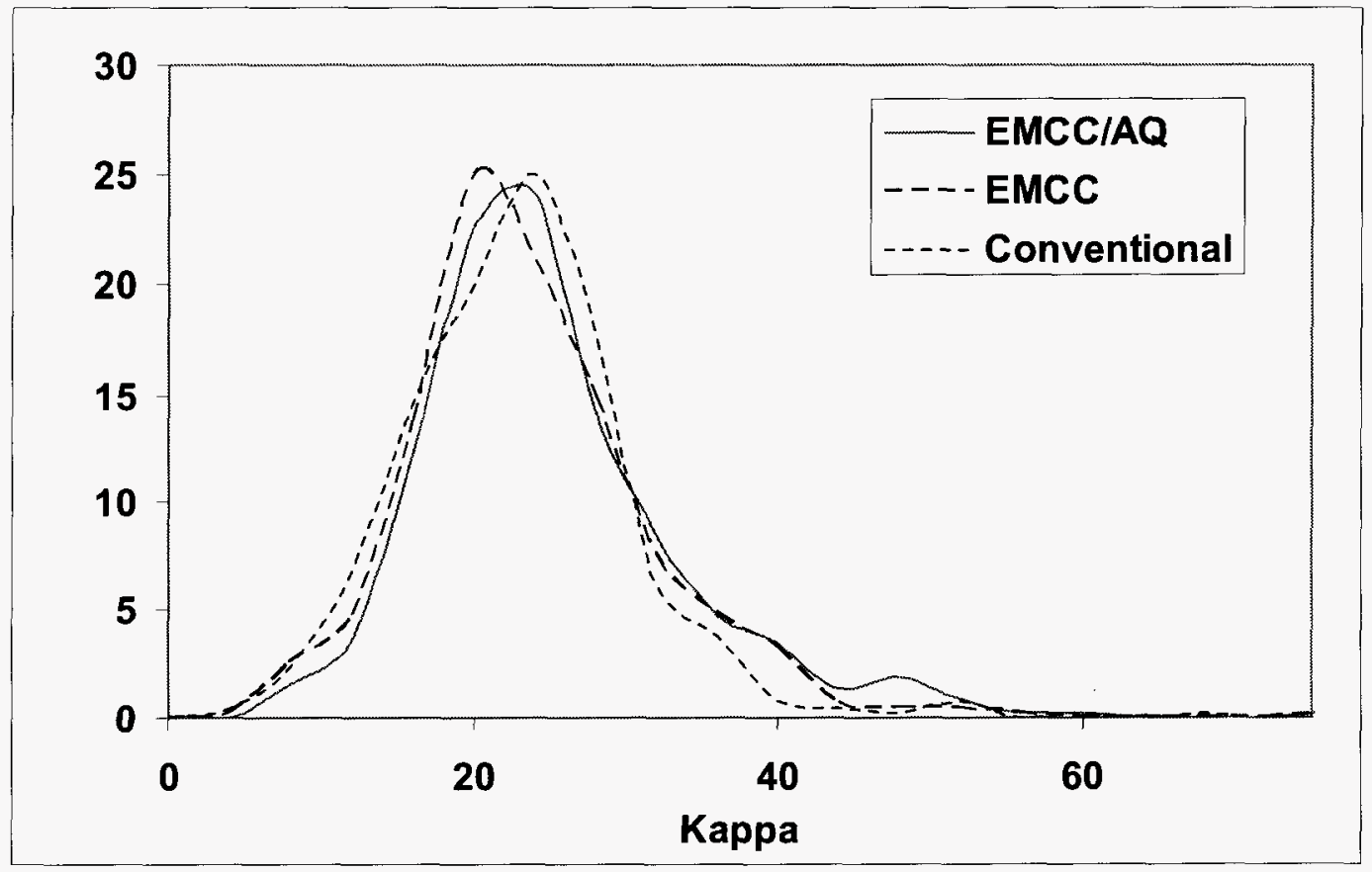

Figure 12. Kappa histograms comparing kappa 22 pulps from different kraft cook types. 


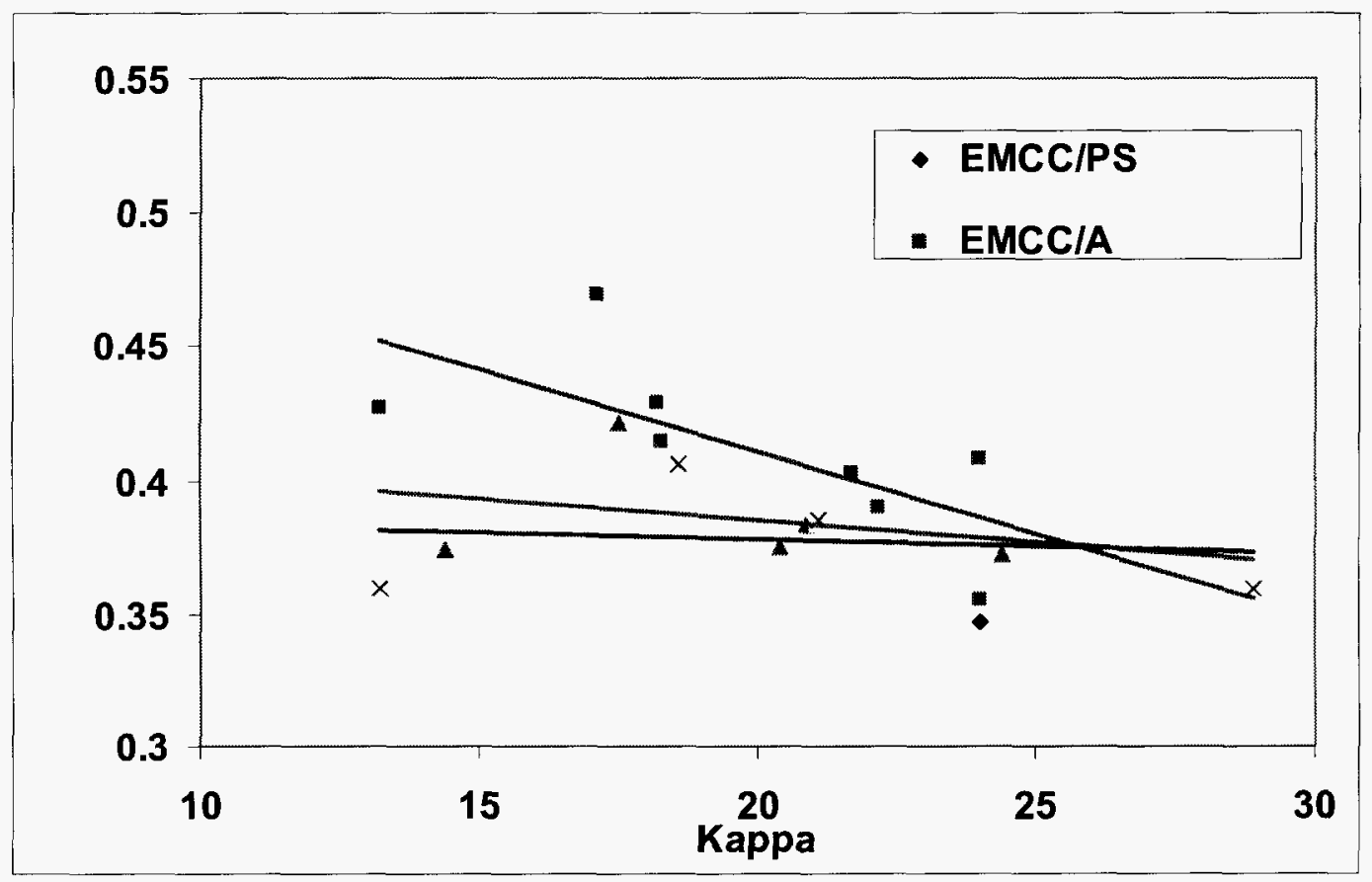

Figure 13. Dependence of coefficient of variation on bulk kappa number for different kraft cook types.

\section{Pulp pre-treatment study}

A pulping study is under way at the Helsinki University of Technology (HUT) to investigate the efficacy of various chip pre-treatments on pulp uniformity. In this study, softwood chips are either pre-steamed, pressure impregnated, or both. The treated chips are then Kraft pulped. Control, untreated, wood chips were also pulped. Table I details the pulps analyzed in this study. The A chips are primarily heartwood chips, which are difficult to impregnate, and the B samples are primarily sapwood chips which have a more open pore structure.

Table I. Pulping Pre-treatments and Kappa of Pulp Used in Uniformity Study

\begin{tabular}{|c|c|c|c|c|}
\hline Sample Name & Pressure & Steaming & Kappa & $\begin{array}{l}\text { Chip Moisture } \\
\text { Content (\%) }\end{array}$ \\
\hline SB A1 & LOW & NO & 34.4 & 34 \\
\hline SB A2 & HIGH & NO & 31.3 & 35 \\
\hline SB A3 & LOW & YES & 31.5 & 30 \\
\hline SB A4 & HIGH & YES & 29.5 & 32 \\
\hline SB B1 & LOW & NO & 36.2 & 30 \\
\hline SB B2 & HIGH & NO & 35.2 & 30 \\
\hline SB B3 & LOW & YES & 34.0 & 33 \\
\hline SB B4 & HIGH & YES & 33.9 & 28 \\
\hline
\end{tabular}


Figure 14 shows the Kappa distribution for the A samples. It can be seen that no pre-treatment, A1, leads to a broader kappa pulp. Steaming the chips or pressure impregnation resulted in fibers in the kappa 40 - 70 range being shifted to close to the mean kappa (low 30s). Neither pretreatment resulted in an increase in the number of low kappa fibers. That is, the distributions did not develop a low kappa tail with pre-steaming or pressure impregnation

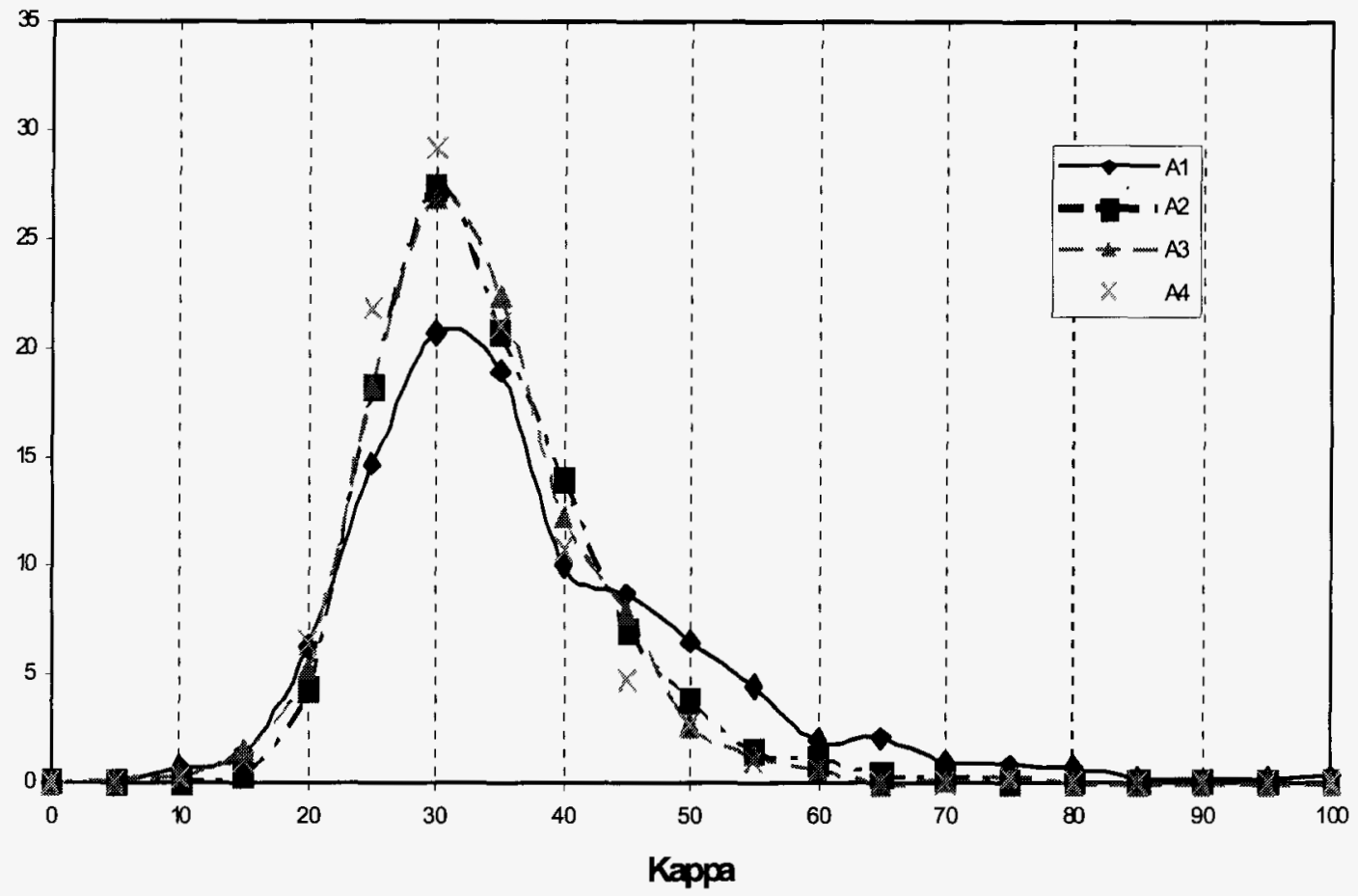

Figure 14. Kappa Distribution of A samples

The distributions show, however, that the pre-steaming and pressure impregnation are equally effective and that once you do one pre-treatment there is little improvement with the second.

Statistics on the kappa distribution are shown in Table II. The statistics reflect what can be seen on the plots; that pre-treating the chips improves the uniformity (lower $2^{\text {nd }}$ and $3^{\text {rd }}$ normalized moments) and that pre-steaming and pressure impregnation are equally effective. 
Table II. Statistics on “A” Samples Kappa Distributions

\begin{tabular}{|c|c|c|c|}
\hline Sample Name & Average Kappa & $\begin{array}{c}\text { Normalized 2 } \\
\text { Moment }\end{array}$ & $\begin{array}{c}\text { Normalized 3 } \\
\text { Moment }\end{array}$ \\
\hline A1 & 34.45 & 39.81 & 1834.14 \\
\hline A2 & 31.31 & 33.85 & 1240.48 \\
\hline A3 & 31.42 & 34.25 & 1275.06 \\
\hline A4 & 29.51 & 31.75 & 1086.76 \\
\hline
\end{tabular}

The B samples showed a similar but much smaller effect of pre-treatment on the pulp uniformity. Figure 15 shows the kappa distribution of the B samples. The distributions all look basically the same.

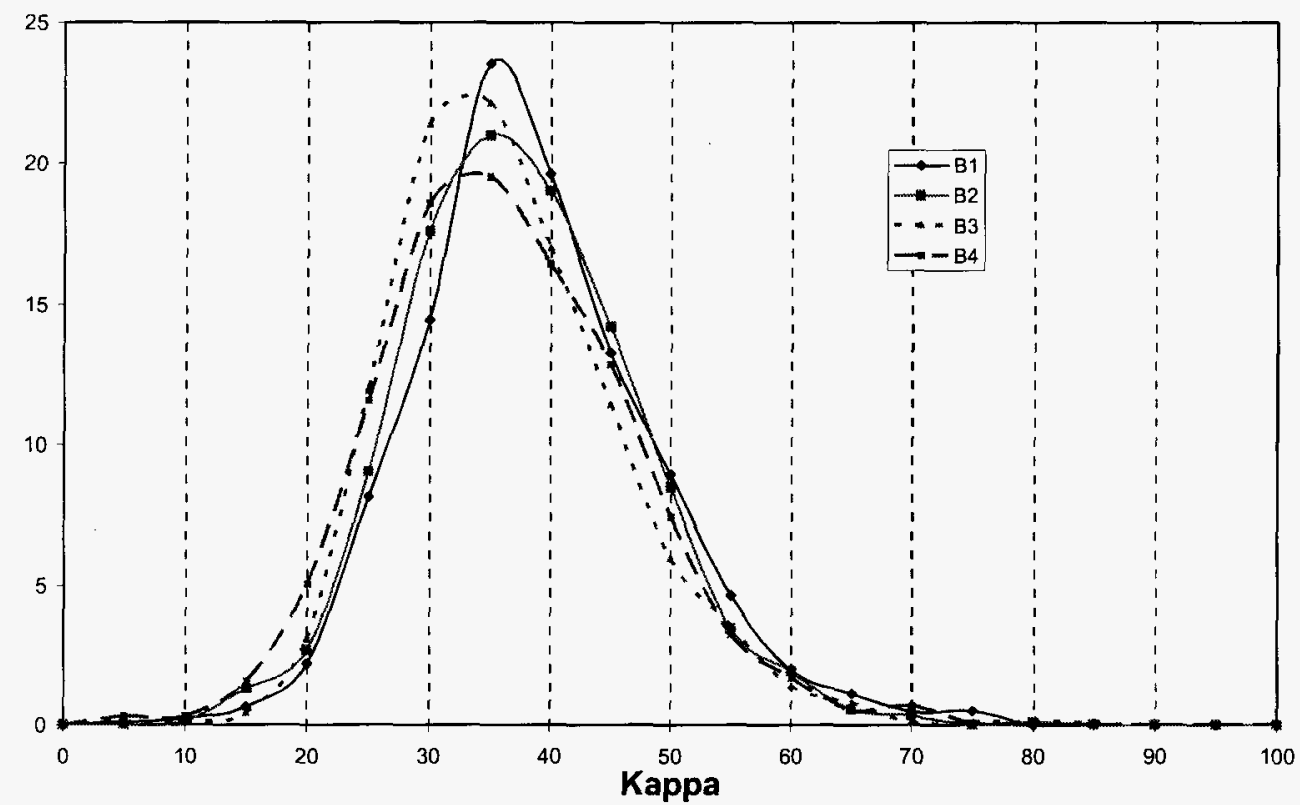

Figure 15. Kappa distributions of B samples

Statistics on the B sample kappa distributions are shown in Table III. Table III suggests that the pre-treated samples had a slightly narrower distribution than the untreated sample (B1). The difference is not near as significant as was observed in the A samples. These kappa distributions have been discussed with Helsinki University of Technology researchers and are consistent with bulk pulp measurements made on these pulps. Complete results of the study will be forthcoming in a joint publication. 
Table III. Statistics on "B" Samples Kappa Distributions

\begin{tabular}{|c|c|c|c|}
\hline Sample Name & Average Kappa & $\begin{array}{c}\mathbf{2}^{\text {nd }} \text { Normalized } \\
\text { Moment }\end{array}$ & $\begin{array}{c}3^{\text {rd }} \text { Normalized } \\
\text { Moment }\end{array}$ \\
\hline B1 & 36.21 & 39.03 & 1631.99 \\
\hline B2 & 35.17 & 37.85 & 1529.23 \\
\hline B3 & 33.97 & 36.69 & 1450.26 \\
\hline B4 & 33.91 & 37.13 & 1489.62 \\
\hline
\end{tabular}

Single fiber kappa measurements on laboratory and commercial pulps

Comparison of kappa distributions between laboratory pulps and commercial pulps indicate that pulp uniformity can be improved through manipulation of cooking parameters and chip quality. Figure 16 compares the kappa distribution of a lab and a commercial pulp and shows the extent of the improvement that may be made under ideal pulping conditions. The nonuniformity existing in the lab pulp, which was prepared from thin chips pulped at a low temperature with high chemical charges and a high liquor to wood ratio, suggests that there is a limitation on achievable uniformity in a kraft cook, but there is an opportunity for improving uniformity in commercial pulps. The commercial pulp is considerably more non-uniform than the laboratory pulp despite having a lower average kappa number.

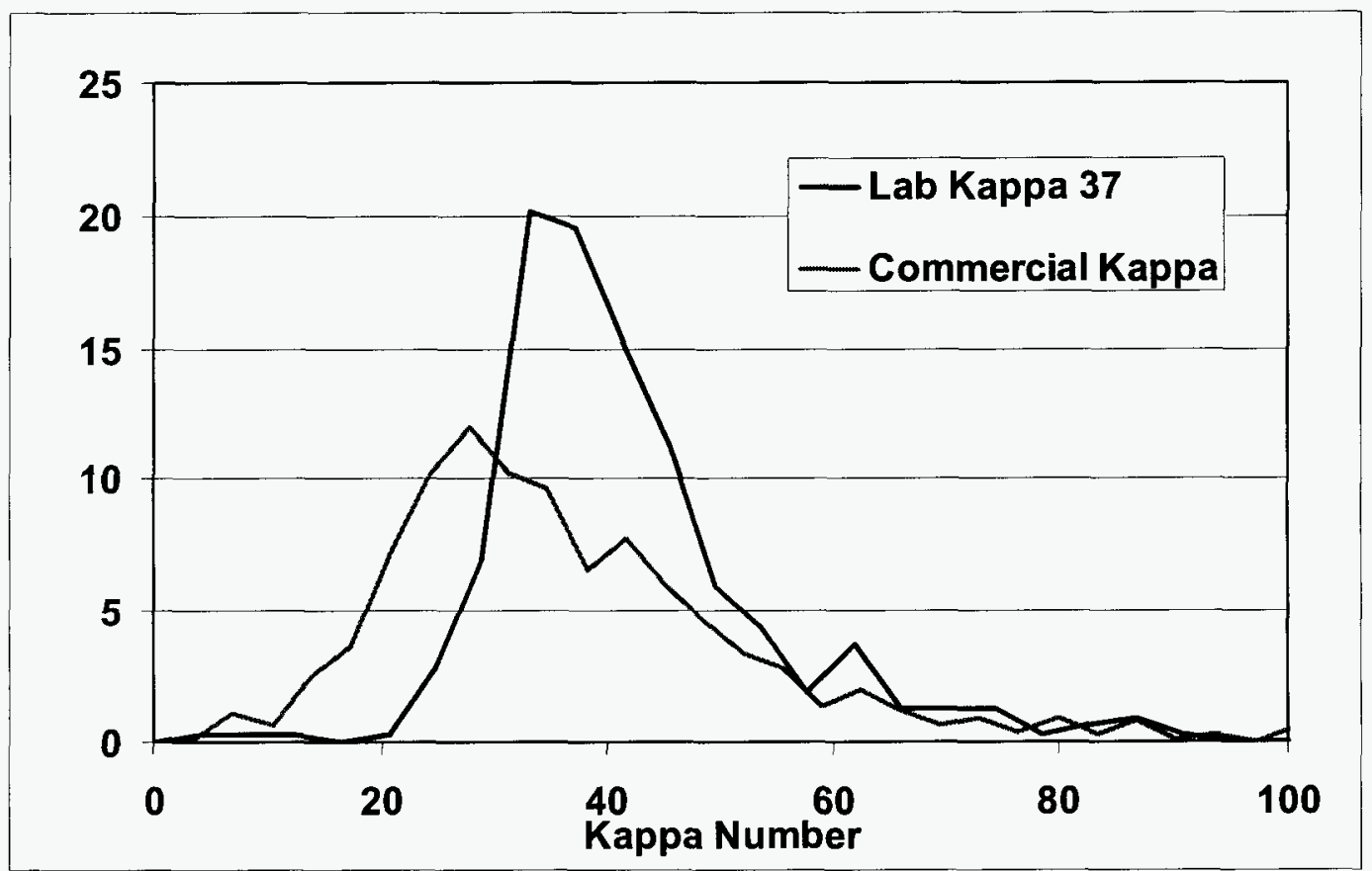

Figure 16. Kappa distribution of a laboratory pulp and a comparable commercially produced pulp. 


\section{M\&D Digester Study}

Recently, two industrial samples were provided with known origin and pulping conditions. Figure 17 represents the kappa distribution obtained from these two samples. The first sample was produced in a batch digester from chips. The second sample was pulped in a continuous M\&D digester from sawdust.

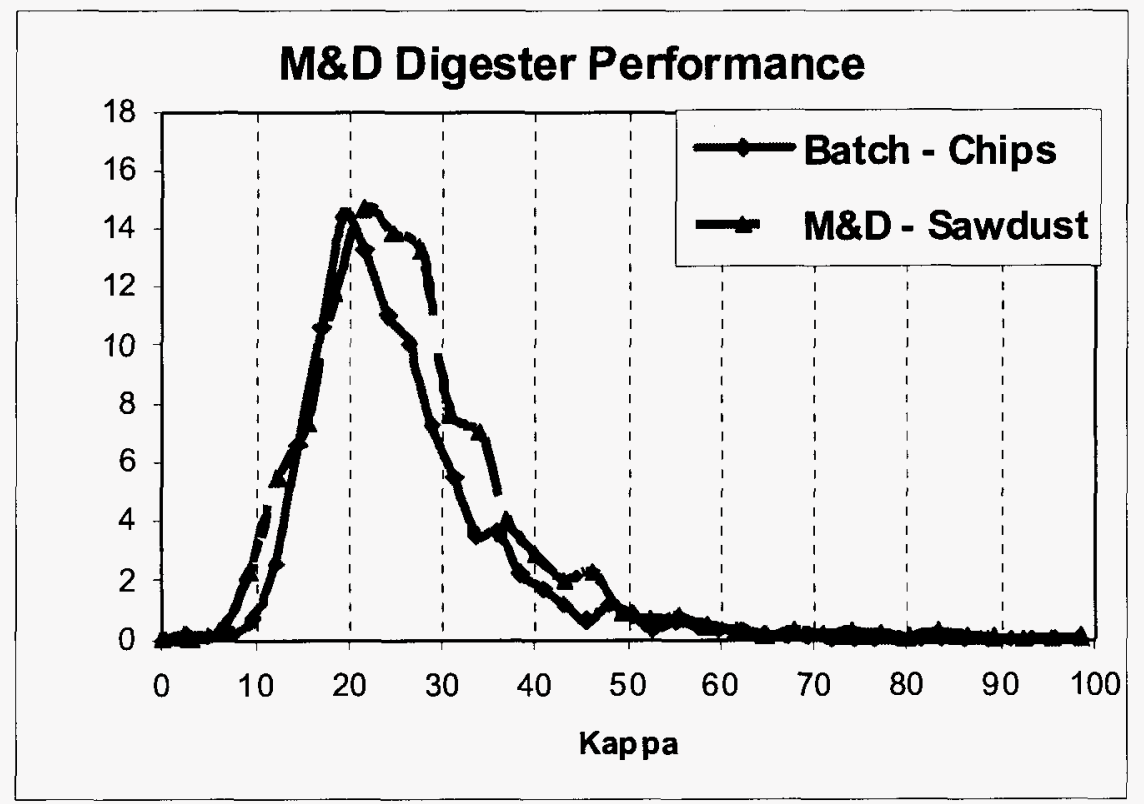

Figure 17. Single Fiber Kappa Distributions For Two Pulping Processes

The first feature of interest in Figure 17 is the greater low kappa shoulder of the M\&D cooked sawdust pulp. This is consistent with relatively over-cooked portions of the high surface area sawdust pulp. The chip pulp, which lacks this low kappa shoulder, presents a more uniform approach to the mean kappa as has been previously measured on similar chip pulps produced in the laboratory. Secondly, note the relatively high kappa tail of the sawdust pulp. While unexpected, a review of the pulping process employed by this manufacturer offers a good explanation for this result. The M\&D digester throughput is extremely high. Cooking time has been reduced in the M\&D digester in order to maximize production at this site. Because of the short cooking time and high packing density of sawdust in the digester, pockets of uncooked raw material render significant portions of high kappa fibers. Additionally, a significant portion of pins has been included in the sawdust pulp from their supplier. These factors, combined with the small over-cooked portion, explain the wider total kappa distribution for the sawdust pulp.

\section{Pin chip study}

Three additional commercial samples have been analyzed. These samples were part of a pin chip addition level trial at a US pulp mill. The first sample represents a pre- 
trial control which consisted of a standard $20 \%$ pin chip addition to the digester. The trial condition consisted of $30 \%$ pin chip addition to the digester. Finally, a second control was run when the pin chip level was believed to have returned to the original $20 \%$. Results from the FKA analysis of these three pulps are reported in Figure 18.

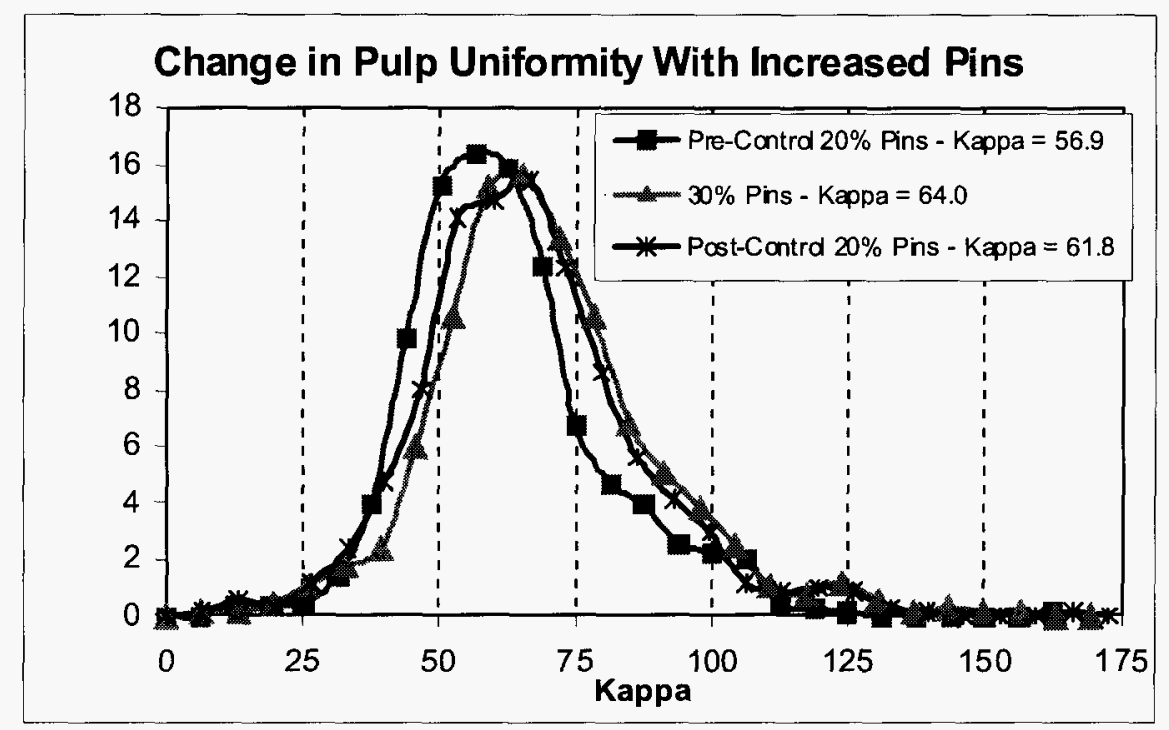

Figure 18. Single Fiber Kappa Distributions For Two Raw Materials

A $10 \%$ increase in pin chips does not represent a significant modification in process conditions. However, several small differences in the single fiber kappa distributions between these three samples are noteworthy. First, the pulp with more pins had the greatest bulk kappa. This result was not expected as increasing the small chip fraction should facilitate liquor impregnation and easier lignin removal given constant cooking conditions. This is most likely due to the inability of this facility to hold the flux of wood added to the digester constant over changing pin levels. This accounts for the high kappa shift of the $30 \%$ pins fraction kappa distribution. Secondly, the $30 \%$ pins fraction reported a narrower peak area than the two controls. While this difference is slight, it would be expected given more uniform liquor impregnation of the enhanced pins pulp. The high kappa shoulders for both the trial condition and post-control correspond well with an unusually high level of rejects measured on these pulps. Finally, the postcontrol sample produced the broadest distribution of all three samples tested. This finding is consistent with post analysis of the pulps which indicated that the material produced immediately after the trial was off-specifications with regards to kappa and most significantly rejects.

\section{Additional single fiber measurements}

The single fiber kappa analyzer instrument has been augmented to make other single fiber measurements. Discussions with various experts on fiber, pulp, and paper properties suggested that a suite of fiber properties would be required to develop useable 
relationships between single fiber measurements and performance of the paper sheet. The research outlined below was not directly supported by the DOE grant but is presented here to provide a complete picture of research related to and initiated by the DOE grant.

Cellulose fiber shape (curl and kink) can now be determined simultaneously with fiber kappa and length in the single fiber analyzer. Fiber shape is defined as a deflection of the main longitudinal axis of the fiber. See Figure 19 for a description of fiber Curl Index.

Figure 19. Curl Index Description

The correlation of fiber shape to paper properties is well understood. Table IV provides an example of the effect of fiber shape on the tensile strength of paper. In general, increased fiber deflections result in less inter-fiber bonding and a weakening of the cell wall structure. Having the unique ability to measure both kappa and shape of individual fibers allows for never-before-made correlations to be investigated between the chemical content and physical structure of papermaking fibers.

Table IV. Significance of Fiber Shape

\begin{tabular}{|l|c|c|}
\hline Material Tested & Curl Index & Tensile Strength, km \\
\hline Un-bleached Handsheets & 0.1 & 7 \\
\hline Un-bleached Handsheets & 0.15 & 5 \\
\hline Un-bleached Handsheets & 0.2 & 3 \\
\hline Un-bleached Handsheets & 0.25 & 1.5 \\
\hline
\end{tabular}


One logical correlation between chemical content and fiber shape is that the fibers containing the most lignin could remain relatively straighter than the delignified fibers. Preliminary results comparing these two properties are reported in Figure 20. For these softwood fibers with an average bulk kappa of 21 , the straight fibers had an average kappa of 72. The highly curled fibers reported an average kappa of 19.

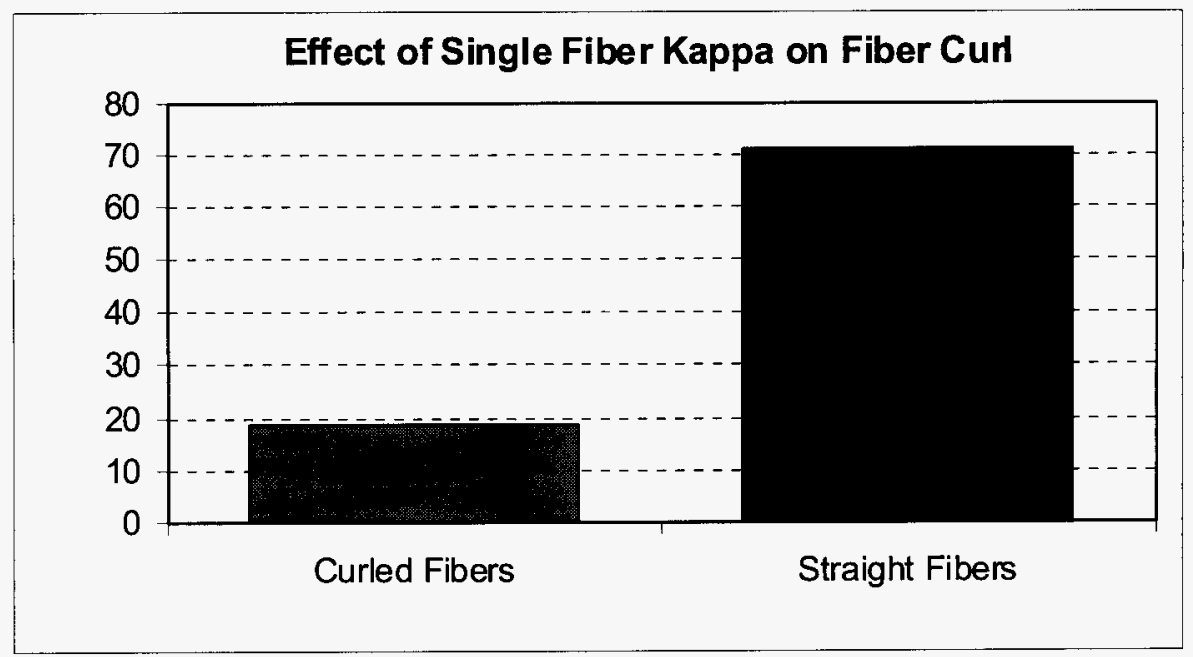

Figure 20. Single Fiber Kappa - Shape Relationship

We have also begun research to use the single fiber analyzer as a tool for measuring single fiber surface charge. This project is born out of the successes achieved with the single fiber kappa project. The analytical equipment from the single fiber kappa project will directly apply to this undertaking. The first stage of this project consisted of an extensive literature review of previous work in the area of surface charge measurements and fluorescence applications relevant to this study. As a result of this search we have identified 26 fluorescent stains that are suitable candidates for the proposed research. Samples of these stains have been acquired and will be screened for their cellulose fiber charge specificity. To facilitate screening of the stains we have obtained pulp fibers from a pulp and paper company that have been surface modified. These pulps are bleached kraft fibers, in which the surface carboxyl content has been modified to four levels. Stains that are specific to surface charge should sorb and fluoresce markedly different on these different pulp fibers. 
Candidate stain evaluations have already begun. Preliminary results using a Zeiss fluorescence microscope and monochrometer have demonstrated marked fluorescence intensity shifts for higher charged fibers. Figure 21 shows the visual intensity difference between carboxylated (red) fibers and non-carboxylated (pink) fibers treated with propidium iodide. Under identical treatment conditions, the higher charged fibers attracted more stain to their surfaces. Similarly, the DNA stain ethidium bromide and membrane potential dye Vybrant DiI have also shown intensity shifts between differently charged fibers. Figure 22 depicts the measured intensity difference between carboxylated and non-carboxylated fibers treated with Vybrant DiI.

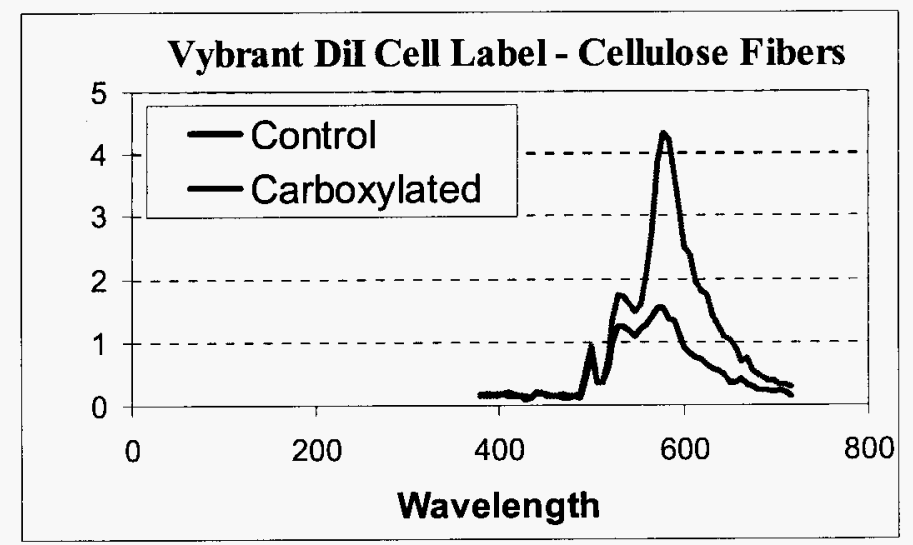

Figure 21. (left). Image of Propidium Iodide Treated Carboxylated (red, right) Fibers and Uncarboxylated (pink, left) Fibers

Figure 22. (right). Fluorescence Emission Spectra of Vybrant Dil Treated Carboxylated and Non-Carboxylated Fibers

Work will continue on this project to develop a single fiber surface charge measurement similar to the single fiber kappa measurement.

\section{Conclusions}

A method has been developed to measure the kappa number of single wood fibers. The method uses the lignin induced red-shift fluorescence of the stain Acridine Orange. The ratio of red to green fluorescence correlates well with kappa number for a given pulp type. An instrument that uses this method has been developed to routinely and easily measure pulp uniformity. The accuracy of the instrument has been confirmed by comparison pulp of uniformities measurements with the flow-through instrument with those made with a static fluorescence microscope. The kappa distributions measured by the new instrument were also compared with those made by a density gradient column with excellent results. Pulp uniformity measurements have been made on many laboratory and commercial pulps. These measurements have lead to a deeper understanding of the nature of pulp uniformity and the relationships between pulping conditions and pulp uniformity. We continue to provide service to mills and research laboratories that want to measure pulp uniformity. 
The technology developed in this research program is being used to make other single fiber measurements such as single fiber surface charger.

\section{Future work}

The DOE grant has expired but research on this sensor continues. We are continue to run samples from pulp mills and are always seeking more samples. Research continues on the surface charge measurement project and a project that relates single fiber kappa to fiber geometry and morphology. A second generation single fiber kappa instrument is almost complete. The new instrument will have better signal to noise which will enable us to measure kappa distributions of higher kappa pulps. We have filed an invention disclosure with the University and that is being processed. Finally, we have begun working with Systematix Controls to develop a commercial single fiber analyzer. Systematix sells automated kappa analyzers and has considerable experience in building commercial instruments that use optical means, light and color, for the analysis.

\section{Acknowledgements}

This project has been supported by several institutions, companies and government agencies. The following are those that have contributed to this research program. The authors gratefully acknowledge their support:

Weyerhaeuser Co., Champion International (now International Paper), Department of Energy Agenda 2020 program, AF\&PA members on the sensor and controls task force, University of Washington Denman Professorship, Pulp \& Paper Research Institute of Canada for help with the flow cell, and Alan Rudie of IPST for providing pulp samples and uniformity data.

\section{References}

1. Tikka, P. and Kovasin, K., "Displacement vs. conventional batch kraft pulping: delignification patterns and pulp strength delivery," Paperi ja Puu 72(8):776 (1990).

2. Tikka, P., MacLeod, J., and Kovasin, K., "Chemical and physical performance of kraft cooking: the impact of process alternatives," Tappi Journal 74(1):140 (1991).

3. Thomson, S. and Gustafson, R., "Effects of kappa number variability on pulp properties," Tappi Journal 83(1):162 (2000).

4. Christie, D., "Chip screening for pulping uniformity," Tappi Journal 70(4):113 (1987).

5. Paulson, J., "Unbleached pulp uniformity in prehydrolysis-kraft pulp from Slash Pine," Svensk Papperstinding 74(13-14):399 (1971).

6. Boyer, B. and Rudie, A. "Measurement of delignification diversity within kraft pulping processes," Tappi Pulping Conference Proceedings, 767 (1995).

7. Liu, Y., Gustafson, R., Callis, J., and McKean, W., "A novel method to measure fiber kappa number," Tappi Journal 82(9):109 (1999).

8. Liu, Y., "Application of secondary fluorescence to measure the kappa number of single fibers," PhD Thesis, University of Washington, 79 (1998).

9. Liu, Y., "Application of secondary fluorescence to measure the kappa number of single fibers," $\mathrm{PhD}$ Thesis, University of Washington, 25 (1998). 
10. Boyer, B., "Measurement of delignification diversity within kraft pulping processes", $\mathrm{PhD}$ Thesis, Institute of Paper Science and Technology, 26(1998).

11. McDonough, T. and Herro, J., "The influence of low-lignin pulping conditions on bleachability," Tappi Journal 65(9):118 (1982). 